high TB prevalence region like India. TST with all its limitation probably will continue to serve a useful purpose. Larger prospective studies are needed in high incidence countries to determine the sensitivity and specificity of IGRAs, their ability to serve as useful rule-out test for active TB.

\section{P13 INTERFERON-GAMMA RELEASE ASSAY (IGRA) CONVERSION, REVERSION AND IMPLICATIONS FOR THE DIAGNOSIS OF LATENT TUBERCULOSIS INFECTION USING A MULTIMODALITY APPROACH: A RETROSPECTIVE, OBSERVATIONAL STUDY WITHIN A CENTRAL LONDON TB CENTRE}

doi:10.1136/thoraxjnl-2011-201054c.13

${ }^{1} \mathrm{M}$ H Almond, ${ }^{1} \mathrm{M}$ O'Donoghue, ${ }^{2} \mathrm{~N}$ Drey, ${ }^{3} \mathrm{~S}$ Seneviratne, ${ }^{4} \mathrm{~A}$ Lalvani, ${ }^{1} \mathrm{M}$ Wickremasinghe, ${ }^{1} 0 \mathrm{M}$ Kon. ${ }^{1}$ Chest and Allergy Department, St. Mary's Hospital, Imperial College NHS Trust, London, UK; ${ }^{2}$ School of Community and Health Sciences, City University, London, UK; ${ }^{3}$ Department of Clinical Immunology, St. Mary's Hospital, Imperial College NHS Trust, London, UK; ${ }^{4}$ Tuberculosis Research Unit, St. Mary's Hospital, Imperial College NHS Trust, London, UK

Introduction and Objectives Accurate diagnosis \& management of latent tuberculosis infection (LTBI) among TB contacts is critical for both the health of infected individuals and prevention of disease transmission. Interferon gamma release assays (IGRAs) measure $\mathrm{T}$ cell release of interferon-gamma following stimulation by antigens not confounded by the BCG vaccination. The current NICE guidelines recommend their use following a positive TST. In addition some centres have moved to a single step IGRA test for LTBI. Our institution adopts a triple investigation approach comprising a chest radiograph (CXR), TST and IGRA on presentation followed by a rescreen if the TST \& IGRA are discordant or if pulmonary contacts are screened prior to 6 weeks. The aim of our study was to evaluate the prevalence of IGRA conversion and reversion in rescreened asymptomatic $\mathrm{TB}$ contacts that attended our centre.

Methods This was a retrospective, observational study carried out at a central London teaching hospital. The study population comprised 593 consecutive, adult TB contacts screened between 1 January 2008 and 31 December 2010. Data were collected through retrospective review of chest radiographs, TST \& IGRA tests.

Results Of 498 asymptomatic TB contacts screened, 460 had both an initial TST and IGRA performed (Abstract P13 figure 1). 81

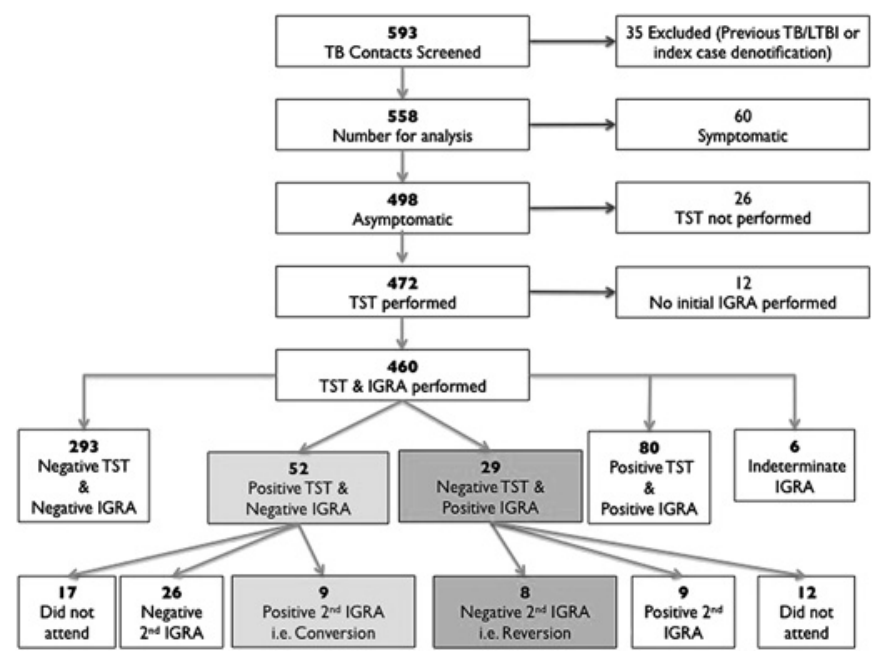

Abstract P13 Figure 1 LTBI Diagnostic Flow showing that an IGRA rescreen can both identify LTBI and reduce the number of false positives.
(17.7\%) contacts had discordant TST \& IGRA results. 52 (64\%) of these discordant cases had a positive TST \& Negative IGRA; these patients would have been discharged under NICE guidelines however, our rescreen revealed $9(17 \%)$ positive $2^{\text {nd }}$ IGRAs that is, conversion. Three of these patients were under 35 and would therefore by eligible for chemoprophylaxis. Twenty-nine (36\%) of the discordant cases had a negative TST and positive IGRA however, $8(28 \%)$ of these IGRAs reverted to negative. It is important to note that if following a single-step IGRA screening protocol (ie, without a rescreen) these cases may have been commenced on chemoprophylaxis unnecessarily (four of these reversion cases were under the age of 35).

Conclusions Our results show that adoption of either a sequential TST + ve/IGRA approach or single IGRA approach can result in a significant number of false negative LTBI diagnoses due to IGRA conversion. Conversely, we have also shown that an IGRA rescreen because of discordant TST/IGRA tests can improve LTBI diagnostic specificity and therefore reduce unnecessary chemoprophylaxis due to the effect of reversion.

\section{P14 COMPARISON OF TWO INTERFERON-GAMMA RELEASE ASSAYS (QUANTIFERON-TB GOLD IN-TUBE AND T-SPOT.TB) IN SCREENING FOR LATENT TUBERCULOSIS INFECTION (LTBI) AMONG HIV-INFECTED ADULTS ATTENDING AN INNER LONDON HIV CLINIC}

doi:10.1136/thoraxjnl-2011-201054c.14

${ }^{1} \mathrm{R}$ F Miller, ${ }^{2} \mathrm{~T}$ Mahungu, ${ }^{1} \mathrm{M}$ Young, ${ }^{1} \mathrm{D}$ Mercey, ${ }^{2} \mathrm{~S}$ Morris-Jones, ${ }^{1} \mathrm{P}$ Benn. ${ }^{1}$ Mortimer Market Centre, Camden Provider Services, Central and North West London NHS Foundation Trust, London, UK; ${ }^{2}$ Department of Microbiology, University College London Hospitals NHS Foundation Trust, London, UK

Background There is a paucity of data on the performance of interferon-gamma release assays (IGRAs) in screening HIV-infected patients for latent tuberculosis infection (LTBI).

Methods Prospective evaluation of two IGRAs [QuantiFERON-TB Gold In-Tube (QuantiFERON) and T-SPOT.TB] for screening HIVinfected patients attending an inner city clinic with a high local prevalence of tuberculosis (TB). For each patient we recorded their gender, ethnicity, country of birth, BCG status and prior treatment for TB, CD4 count, plasma HIV load and receipt of antiretroviral therapy (ART). All patients had both IGRAs (results = positive, negative, or indeterminate) and a chest radiograph.

Results Of 117 patients, 91 (78\%) were men, 48 (41\%) were white British, 29 (24.8\%) black African, 21 (18\%) European, 7 (6\%) South American and $12(10 \%)$ from other ethnic groups. Individuals born outside the UK had been resident in the UK for a median of 10 years (range 3-45). 91/117 (78\%) had previously received BCG and $14 / 117(12 \%)$ had been treated for TB a median of 9.5 years ( range 3-13) previously. Of 106 (90.6\%) receiving ART, 105 (99\%) had an undetectable plasma HIV load) and the median CD4 count was 550 (range 30-1250). Among those not receiving ART the median (range) plasma HIV load and CD4 count was 21000 copies/ $\mathrm{ml}(590-160000)$ and 520 (250-950) respectively. IGRA results are shown in the Abstract P14 table 1. Overall there was good concordance $(92.3 \%)$ between the two IGRAs. Among 14 patients previously treated for TB, $10(71.4 \%)$ had previously received BCG; $4 / 14(28.6 \%)$ had a positive IGRA (3/4 both IGRAs were positive, 1) 4 QuantiFERON was positive). Of those previously treated for TB with negative IGRAs $(\mathrm{n}=10)$ all had an undetectable HIV viral load, 9/10 had been on ART $>2$ years and median CD4 count was 670 (range 180-1250). Five other patients with CD4 $<200$ had negative IGRAs; all had previously received BCG. Mantoux testing, done in 2 of the 5 , was negative. 